\title{
SYSTEMATIC ASTROMETRIC ERRORS IN PULSAR TIMING
}

\author{
L. FAIRHEAD \\ Bureau des Longitudes, 77 Avenue Denfert-Rochereau \\ 75014 Paris, France \\ and \\ Department of Astronomy, University of California \\ Berkeley, CA 94720, USA
}

\begin{abstract}
A new analysis of the timing data acquired on the fast pulsar PSR1937+214 is presented. Parameters are evaluated with various models based on two ephemerides, two atomic time scales and two TT-TB time transformations. Comparisons are carried out with results from other programs. We provide evidence that systematic errors induced by the model adopted are 5 to 10 times larger than the formal uncertainties calculated by the fitting procedure. Great care must thus be taken when using results from different millisecond pulsars timing programs for accurate astrometric purposes
\end{abstract}

\section{Introduction.}

Soon after the discovery of the millisecond pulsar PSR1937+214 (Backer et al , 1982), it was realized that beyond its exceptional significance for astrophysics, this new celestial object could also contribute significantly to solar system dynamics studies, to the long-term stability of atomic clocks and to astrometry. The interest grew when additional millisecond pulsars were discovered to form a new class of pulsars.

The use of millisecond pulsars for astrometry will be fully established with the combination of timing and VLBI observations of these objects. The unprecedented astrometric precision of \pm 0.2 milli-arcseconds for the equatorial coordinates of PSR1937+214, estimated by Rawley et al (1988) from timing observations, prompted us to assess possible sources of systematic errors.

We developed a new data analysis package adopting this astrometric approach and flexible enough to accomodate various Earth ephemerides, atomic time scales and TT-TB transformations for the assessment of systematic errors.

\section{Observations and Reduction Method.}

\subsection{OBSERVATIONS}

The data we have analysed were taken at the 305-m radio-telescope in Arecibo from November 1982 to October 1984 by a group from University of California at Berkeley and Princeton University (full detals are in Davis et al, 1985). 
We were also provided by Dr. J.Taylor of Princeton with more precise data from Arecibo spanning seventeen months from November 1987 to March 1989. This data set was analysed using our pulsar timing package and compared to the results from the Princeton package.

Pulse arrival times are measured relative to the station's UT. For the analysis, it is necessary to transform this scale to a uniform time scale by first transforming UT to a national or international standard atomic time scale (TA), then to a terrestrial time (TT) and finally to a Barycentric Time scale (TB). TB will also be substituted for time in the ephemeris used to provide the coordinates of the Earth. Several national standards atomic time scales (NBS, NRC, USNO, PTB ...) as well as their combination by the BIPM (TAI, TTBIPM) (Guinot, 1988) are available. Discrepancies exist between these time scales such as an annual variation of $\approx 300-400 \mathrm{~ns}$ between TA(USNO) and TA(PTB) (Guinot, private communication). The transformation from a national standard atomic time scale to the TT scale reduces to the addition of the last term of equation (6) in Guinot (1986) which depends on the Earth orbital velocity and the clock geocentric location. Finally, there are two approaches to convert from TT scale to the TB scale. One is based on a numerical "time ephemeris" as described by Hellings (1986) and the other is an analytical formula (e.g. Fairhead and Bretagnon, 1989). The consequences of these two different approaches will be discussed below.

It is important to realize that the TB scale constructed according to such a scheme will not be unique and should be designated by the name of the atomic time scale initially used in the time chain described in section 2 , such as TB(USNO) or TB(BIPM).

\subsection{DATA ANALYSIS}

The analysis consists in calculating precisely the changes in the propagation time of an electro-magnetic signal from the pulsar to the radio-telescope. These changes depend on the time-dependent position and velocity of the telescope with respect to the centre of the Earth, the position and velocity of the Earth in a reference frame centered on the Solar System Barycentre (SSB), the position and proper motion of the pulsar with respect to the SSB as well as the delay on an electro-magnetic signal traveling in the solar system gravitational field, the interstellar dispersion and the transformation from terrestrial time to coordinate time. The effects of the pulsar strong gravitational field need not be included in this analysis of PSR1937+214 data as it is an isolated pulsar.

The computation of the propagation delay is carried out in the framework of General Relativity theory in isotropic coordinates. The corresponding metric can be found for example in Hellings (1986). The following simple formulation for the transformation from pulse arrival time to pulse emission time is not complete but will suit our illustration needs (the full formulation is in Hellings(1986)):

$$
T_{N}=t_{N}^{o b s}-\frac{R_{0}}{c}+\vec{k} \cdot \vec{r} / c+\Delta_{r e l}+\Delta_{T B}+k D M / \nu^{2}
$$

where $t_{N}^{o b s}$ is the arrival time of a pulse measured at the radio-telescope and $T_{N}$ is its emission time at the pulsar. $R_{0}$ is the pulsar distance from the SSB, $\vec{k}$ is a unit vector from the SSB in the direction of the pulsar at time $t_{N}^{o b s} . \vec{r}$ is the position of the radio-telescope with respect to the SSB at time $t_{N}^{o b s} . \Delta_{r e l}$ is the relativistic delay on the signal. $\Delta_{T B}$ is the transformation from Terrestrial Time, TT, to coordinate time, TB. The delay $k D M / \nu^{2}$ is caused by interstellar medium dispersion, with $\nu$ the observation frequency Doppler-shifted to the SSB by taking the velocity of the telescope into account. Once the measured arrival times $\left(t_{N}^{o b s}\right)$ have been transformed into emission times $\left(T_{N}\right)$ using the model of equation (1), one has:

$$
T_{N}-T_{0}=N P
$$


where $T_{0}$ is the emission time of an arbitrary pulse. $N$ is the number of pulses emitted by the pulsar between $T_{0}$ and $T_{N}$ and $P$ is the period of the pulsar at $T_{N} . P$ is modeled by:

$$
P=P_{0}+\frac{1}{2} \dot{P}_{0}\left(T_{N}-T_{0}\right)
$$

where $P_{0}$ and $\dot{P}_{0}$ are the period and its first derivative with respect to time at $T_{0}$.

As the a priori values for all the parameters of the model are approximate, equation (2) will not be verified exactly and one has:

$$
T_{N}-T_{0}=(N+\delta) P \quad-0.5<\delta<0.5
$$

The prefit residuals $\delta P$ and the partial derivatives of $T_{N}-T_{0}$ with respect to the parameters of the model are then fitted using a linear least-square procedure to determine new parameters for the model, such as the period and period derivatives of the pulsar, its position, or the orbital parameters of the Earth.

\subsection{REFERENCE SYSTEMS AND EPHEMERIDES}

2.3.1 The Ecliptic Reference Frame Pulsar timing data are essentially sensitive to the Earth orbital motion and to the pulsar position as shown by the leading term $\frac{\vec{k} \cdot \vec{r}}{c}$ in equation (1). The ephemeris providing the Earth orbital motion also defines the ecliptic plane. Consequently, the natural coordinate system for the pulsar position and proper motion is the ecliptic reference frame of the ephemeris adopted. We have chosen this new astrometric approach, over the classical use of the equatorial system, to avoid a source of uncertainty which is the transformation from ecliptic to equatorial coordinates. Positions of the line of the equinox can differ by $0.1^{\prime \prime}$ and values of the obliquity by $0.03^{\prime \prime}$ as given by different ephemerides and recommended by the IAU (Standish 1982, Bretagnon private communication).

2.3.2 Ephemerides A high accuracy ephemeris is needed since timing measurement uncertainty for PSR1937+214 is at the $1 \mu$ s level corresponding to $300 \mathrm{~m}$ on the orbit of the Earth. We have used two ephemerides in this analysis: the JPL ephemeris DE200 (Standish 1982) and an ephemeris developped at the BdL (LeGuyader, 1989). Finally, we compared our results to those obtained using the CfA ephemeris PEP740R

The actual orbit of the Earth is unique but its representations by these three ephemerides will differ at some level. The superposition of the three representations of the Earth orbit will lead to possible non-alignments, rotations rates and linear drifts between the ephemerides reference frames. For example, there is a limit of 0.06 milli-arcsecond per year in the rotation between the DE200 and PEP740R reference frames (Standish as quoted by Rawley, Taylor and Davis (1988)).

\section{Results}

The results of several solutions on the 1982-84 data, combining two ephemerides (BDL, DE200), three atomic time scales (USNO, TAI, BIPM) along with the two TT-TB transformation formulæ are presented in tables I and II. The residuals $-P \delta$ were fitted by a weighted least square procedure for the following parameters: the period $P_{0}$ and its first derivative $\dot{P}_{0}$, the ecliptic coordinates, $\lambda_{0}$ and $\beta_{0}$, the proper motion, $\mu_{\lambda_{0}}$ and $\mu_{\beta_{0}}$, at epoch J2000 and the epoch of origin $T_{0}$. Post-fit residuals are similar for each solution and were 
tested for normality by using the $\chi^{2}$ test and for goodness of fit (Bevington 1969). In the result tables, the normalized $\chi^{2}$ and the normalized goodness-of-fit are included and denoted by $\chi^{2}$ and G, respectively. The root-mean-square (r.m.s.) for the post-fit residuals of each solutions is also indicated.The degree of freedom in our analysis is 188 such that $\chi^{2} \approx .3$ corresponds to a high confidence level (larger than $99 \%$ ) for the gaussian distribution of the post fit residuals and $\mathrm{G} \approx 1$ indicates a good fit of our model to the data points. Hence, a priori measurements uncertainties used in the fit are realistic.

\begin{tabular}{cccc}
\hline Parameter & BDL/BIPM & BDL/USNO & BDL/TAI \\
\hline$P_{0}(\mathrm{~ms})$ & $1.557806448862(5)$ & $1.55780644886354(5)$ & $1.55780644886286(5)$ \\
$\dot{P}_{0}\left(10^{-19} \mathrm{~s} \mathrm{~s}^{-1}\right)$ & $1.05126(1)$ & $1.05128(1)$ & $1.05127(1)$ \\
$\lambda_{0}(J 2000.0)$ & $301^{\circ} 58^{\prime} 23^{\prime \prime} .7842(12)$ & $301^{\circ} 58^{\prime} 23^{\prime \prime} .7843(12)$ & $301^{\circ} 58^{\prime} 23^{\prime \prime} .7843(12)$ \\
$\beta_{0}(J 2000.0)$ & $42^{\circ} 17^{\prime} 48^{\prime \prime} .3145(2)$ & $42^{\circ} 17^{\prime} 48^{\prime \prime} .3145(2)$ & $42^{\circ} 17^{\prime} 48^{\prime \prime} .3146(2)$ \\
$\mu_{\lambda_{0}}\left(\mu \mathrm{as} \mathrm{y}^{-1}\right)$ & $-381(52)$ & $-372(52)$ & $-395(52)$ \\
$\mu_{\beta_{0}}\left(\mu\right.$ as y $\left.{ }^{-1}\right)$ & $-676(121)$ & $-672(121)$ & $-685(121)$ \\
$T_{0}(\mathrm{JD})$ & 2445303.27316791 & 2445303.27316791 & 2445303.27316791 \\
r.m.s. $(\mu \mathrm{s})$ & 0.970 & 0.985 & 0.974 \\
$\chi^{2}$ & 0.30 & 0.42 & 0.31 \\
$\mathrm{G}$ & 0.85 & 0.88 & 0.86 \\
\hline
\end{tabular}

Table I : Parameters obtained for PSR1937+214 using the BdL ephemeris and 3 different atomic time scales (BIPM= atomic time from the BIPM (Guinot, 1988), USNO= atomic time for the US Naval Observatory (A1), TAI= Atomic time as given by the BIH). The analytical transformation from TT to TB was used in all 3 solutions.

\begin{tabular}{cccc}
\hline Parameter & DE200/BIPM & DE200/USNO & DE200/USNO/JPL \\
\hline$P_{0}(\mathrm{~ms})$ & $1.55780644886286(5)$ & $1.55780644886354(5)$ & $1.55780644886288(5)$ \\
$\dot{P}_{0}\left(10^{-19} \mathrm{~s} \mathrm{~s}^{-1}\right)$ & $1.05127(1)$ & $1.05129(1)$ & $1.05129(1)$ \\
$\lambda_{0}(J 2000.0)$ & $301^{\circ} 58^{\prime} 23^{\prime \prime} .6715(12)$ & $301^{\circ} 58^{\prime} 23^{\prime \prime} .6717(12)$ & $301^{\circ} 58^{\prime} 23^{\prime \prime} .6716(12)$ \\
$\beta_{0}(J 2000.0)$ & $42^{\circ} 17^{\prime} 48^{\prime \prime} .3371(2)$ & $42^{\circ} 17^{\prime} 48^{\prime \prime} .3372(2)$ & $42^{\circ} 17^{\prime} 48^{\prime \prime} .3372(2)$ \\
$\mu_{\lambda_{0}}\left(\mu\right.$ as y $\left.{ }^{-1}\right)$ & $-359(52)$ & $-349(52)$ & $-375(52)$ \\
$\mu_{\beta_{0}}(\mu$ as y & & -1 \\
$T_{0}(\mathrm{JD})$ & $-669(121)$ & $-664(121)$ & $-683(121)$ \\
r.m.s. $(\mu \mathrm{s})$ & 2445303.27316791 & 2445303.27316791 & 2445303.27316791 \\
$\chi^{2}$ & 0.969 & 0.984 & 0.974 \\
$\mathrm{G}$ & 0.29 & 0.22 & 0.27 \\
\hline
\end{tabular}

Table II : Parameters obtained for PSR1937+214 by using the JPL ephemeris DE200, two different atomic time scales (BIPM= atomic time from the BIPM (Guinot, 1988), USNO= atomic time for the US Naval Observatory (A1)) and the numerical TT to TB transformation (denoted by JPL in the column heading, the other 2 solutions used the analytical formula). 


\begin{tabular}{cccc}
\hline Parameter & Princeton & BdL & Berkeley \\
\hline$P_{0}(\mathrm{~ms})$ & $1.557806467568084(12)$ & $1.557806467568084(13)$ & $1.557806467573990(70)$ \\
$\dot{P}\left(10^{-19} \mathrm{~s} \mathrm{~s}^{-1}\right)$ & $1.051149(33)$ & $1.051178(31)$ & $1.050923(35)$ \\
$\alpha(J 2000.0)$ & $19^{h} 39^{m} 38^{s} .560220(4)$ & $19^{h} 39^{m} 38^{s} .560217(4)$ & $19^{h} 39^{m} 38^{s} .562237(3)$ \\
$\delta(J 2000.0)$ & $21^{\circ} 34^{\prime} 59^{\prime \prime} .14177(13)$ & $21^{\circ} 34^{\prime} 59^{\prime \prime} .14184(12)$ & $21^{\circ} 34^{\prime} 59^{\prime \prime} .25119(10)$ \\
r.m.s. $(\mu \mathrm{s})$ & 0.381 & 0.377 & 0.444 \\
\hline
\end{tabular}

Table III : Analysis of the 1987-1989 data set by the various timing packages. All parameters have been estimated for epoch $=2447362.67855354$ JD.

Finally, we have analysed the 1987-89 data using the JPL ephemeris DE200 and the TAI time scale fitting for the following parameters: the period $P_{0}$ and its first derivative $\dot{P}_{0}$, the equatorial coordinates, $\alpha_{0}$ and $\delta_{0}$ at epoch J2000 and the epoch of origin $T_{0}$. Results from the Princeton timing package, the Berkeley timing package and ours on the same data are given in table III. The position determined with the Berkeley program was transformed to J2000. using the procedure described in Murray (1989). The correlation matrix from our analysis is in table IV and shows that none of the parameters are correlated.

\begin{tabular}{r|rrrr} 
& \multicolumn{1}{|c}{$P_{0}$} & \multicolumn{1}{c}{$\dot{P}$} & \multicolumn{1}{c}{$\alpha$} & \multicolumn{1}{c}{$\delta$} \\
\hline$P_{0}$ & 1.00 & 0.57 & -0.02 & 0.41 \\
$\dot{P}$ & 0.57 & 1.00 & -0.11 & 0.76 \\
$\alpha$ & -0.02 & -0.11 & 1.00 & -0.23 \\
$\delta$ & 0.41 & 0.76 & -0.23 & 1.00
\end{tabular}

Table IV : Correlation matrix for the BdL fit on the 1987-89 dataset.

\section{Discussion}

The different solutions were compared with each other and table $\mathbf{V}$ gives the differences between the various parameters.

From the values of the r.m.s., the $\chi^{2}$ and the goodness of fit given by tables I and II, we consider that all the solutions on the 1982- 84 data are statistically equivalent . The choice of ephemeris, atomic time scale or TT to TB time transformation does not affect the r.m.s. or the goodness of fit for this set of data.

\begin{tabular}{c|ccc} 
& USNO/BIPM & BDL/DE200 & PEP740R/DE200 \\
\hline$\Delta P_{0}(\mathrm{fs})$ & $0.69(14 \sigma)$ & $0.01(<1 \sigma)$ & $6(50 \sigma)$ \\
$\Delta \dot{P}\left(10^{-19} \mathrm{~s} \mathrm{~s}^{-1}\right)$ & $0.00002(2 \sigma)$ & $0.00001(1 \sigma)$ & $0.0002(6 \sigma)$ \\
$\Delta \alpha\left(^{s}\right)$ & $0.000009(1 \sigma)$ & $0.000018(2 \sigma)$ & $0.002(500 \sigma)$ \\
$\Delta \delta\left(^{\prime \prime}\right)$ & $0.00001(<1 \sigma)$ & $0.001(5 \sigma)$ & $0.11(1000 \sigma)$
\end{tabular}

Table V : Differences between pulsar parameters when using different models.

The only significant difference between Taylor's result and ours on the same data is a $1 \sigma$ difference in the period derivative $\dot{P}$ whereas the only difference in the models used is the UTC time scale used (UTC(NIST) being used by Taylor and UTC by us). This difference in $\dot{P}$ would be expected to reveal a quadratic term in UTC(NIST)-UTC.Such a quadratic 
term is indeed present in UTC(NIST)-UTC over the time interval in question and with the right order of magnitude to explain the $\dot{P}$ difference (1.2 $\mu$ s amplitude).

The r.m.s. given by Taylor on the 1987-1989 data is equivalent to ours but was calculated with totally independent software except for the ephemeris used. We can thus consider that the rms of $\approx .36 \mu$ s truly characterises that data and that the precision may be limited by receive noise, instabilities in the atomic time or the ephemeris used. We have also determined that with these high quality data we are approaching the precision level of double precision real numbers on most computers and that special steps (such as treating julian dates as integer and fractionnal day numbers) have to be taken to ensure that this limit does not hinder us.

\subsection{OBSERVED DIFFERENCES IN THE PERIOD}

The use of different time scales (BIPM,USNO) in our solutions mainly affects the value of the pulsar period $P$ as shown by table VI. The difference $\Delta P \approx 7 \times 10^{-16} s$ is not compatible with the formal error given by the least-square fit $\left(\sigma \approx 5 \times 10^{-17} s, \Delta P=14 \sigma\right)$. This is explained by a linear drift of $4 \times 10^{-13} \mathrm{ss}^{-1}$ between these two time scales, revealed by a direct comparison of these two time scales. This linear drift is explained by the difference in definition between the two time scales. The BIPM time scale is supposed to be exact and to realize the S.I. second at the $10^{-13} \mathrm{~s}$ level whereas the USNO timescale is required to be stable.

The difference in the parameters when using a numerical or an analytical TT-TB transformation is insignificant on the 1982-84 data as shown by the comparison of the solutions designated by DE200/USNO and DE200/USNO/JPL in table II. By making a direct comparison between the two procedures (Fairhead and Bretagnon, 1989) we have calculated that the periods determined with these two procedures will only differ by $1.5 \times 10^{-17} \mathrm{~s}$ which is the present formal error for the period (table III).

The differing values for the period obtained by the Berkeley program and ours can be explained by a Doppler effect on the period due to the relative velocity of the Earth orbit between one ephemeris and the other (DE200 and PEP740R). The $6 \times 10^{-15} \mathrm{~s}$ difference in the values of the period corresponds to a relative velocity of $35 \mathrm{~km} \mathrm{y}^{-1}$ in the pulsar direction.

This relative velocity is explained by the different values used in the ephemerides for the masses of the outer planets. Over short periods of time (such as the time interval spanned by the data analyzed), the variation of the Sun orbit around the barycentre of the solar system caused by the different values of the masses will appear as a linear drift in the position of the Earth between the two ephemerides. We have determined this linear velocity using a simple ephemeris of the Sun position with respect to the solar system barycenter and by substituting for the masses of the outer planets, thevalues recommende by the I.A.U. and those adopted in the JPL ephemrides. We thus find a linear drift in the motion of the Sun between the two ephemerides of $30 \mathrm{~km} \mathrm{y}^{-1}$ in the direction $\alpha=18^{h} 28^{m}, \delta=0^{\circ}$ for the two years spanned by the data (1982-1984). This leads to a linear drift in the direction of the pulsar, of $25 \mathrm{~km} \mathrm{y}^{-1}$. This is of the order of magnitude that is needed to explain the Doppler shift in the periods determined with respect to the two ephemerides.

\subsection{OBSERVED DIFFERENCES IN PSR1937+214 COORDINATES.}

The use of the different ephemerides BDL and DE200 (using the same atomic time scale) mainly affects the position of the pulsar. The differences in coordinates $\left(0.000015^{s}\right.$ in $\alpha$ and $0.001^{\prime \prime}$ in $\delta$ ) are marginally larger than the formal uncertainties ( $2 \sigma$ and $4 \sigma$, respectively). An annual periodic difference in the position of the Earth will be absorbed in the position 
of the pulsar by the least-square-fit procedure. This is because the position of the pulsar and the position of the Earth are correlated by the leading term $\frac{\vec{k} \cdot \vec{r}}{c}$ in equation (1). A difference of $700 \mathrm{~m}$ in $\vec{r}$ will correspond to the observed difference in the position of the pulsar of $0 . " 001$. The direct comparison of the two ephemerides, BDL and DE200, shows such a difference over the two years of data, figure (3). An absolute difference of $700 \mathrm{~m}$ corresponds to a relative difference of $10^{-9}$ in the position of the Earth. The eccentricity of the Earth which creates an annual term in the position of the Earth has a relative uncertainty of $10^{-9}$ in the BDL ephemeris (Bretagnon, private communication). This could explain the discrepancy in the two ephemerides.

The differences in PSR1937+214 positions between the Berkeley results and ours could be explained by a rigid rotation between the two reference systems in which the coordinates are determined. The reference frame used in the Berkeley package is the one defined by the PEP740R ephemeris which is aligned onto the reference frame of PEP311, a previous ephemeris developed at the CfA for epoch 1982. The reference frames of these two ephemerides drift by $\sim 5$ milliarcsecond $y^{-1}$ because of the different values for the mean motion of the Earth in the two ephemerides (Chandler, private communication). The reference frame of our program is the one inherent in DE200 which has been aligned onto the J2000 reference frame (Standish, 1982).

Bartel et al (1985) and Backer et al (1985) have studied the rotations between the reference systems of the VLA and those of the different ephemerides available form the CfA and the JPL by comparing pulsar timing and interferometric positions. Thus, Bartel et al provide an epoch dependent rotation that best aligns PEP311 to DE118. We have used this rotation plus the rotation matrix between DE118 and DE200 given by Standish (1982) to transform the PEP740R coordinates for PSR1937+21 to the DE200 reference frame but differences of $\Delta \alpha=0.0009^{s}, \Delta \delta=0.0017^{\prime \prime}$ still remain. These differences in coordinates could be explained by a yearly periodic difference between the two ephemerides of $\sim 10 \mathrm{~km}$ or, as seems more likely, by uncertainties in the rotations between the various reference frames and in the epoch-dependency of these rotations.

\section{Conclusion}

We have shown that the parameters determined from the analysis of timing data of one fast pulsar are very sensitive to the choice of ephemerides and atomic time scale used in the analysis. Important differences in the value of the period $(14 \sigma$ and $45 \sigma)$ can be explained respectively by a linear drift of some $4 \times 10^{-13} \mathrm{~s} / \mathrm{s}$ between two atomic scales used and by a linear drift of $35 \mathrm{~km} \mathrm{y}^{-1}$ in the Earth orbit between the two independent ephemerides (DE200 and PEP740R). Differences in the pulsar position could be explained by annual periodic differences of some 700 meters between two ephemerides and by a rotation $(0.19$ " in that region of the sky) between the dynamical reference systems inherent to the two ephemerides. Furthermore, when comparing the Princeton results and ours, we have seen that the quality of the present data is so good that irregularities in the realisations of the UTC time scales used in the analysis are greater than the intrinsic precision of these timing data. One must therefore be very specific about which ephemeris or time scale is used in the analysis when using millisecond pulsar timing data for accurate astrometric applications. One can easily lose all the benefits of these high-precision data because of systematic errors.

The analysis of timing data from many fast pulsars should enable us to decorrelate all these effects and to better constrain atomic time scales on a long time basis ( $>1$ year). Furthermore, they will be useful to compare the different available ephemerides of the Earth and to determine the rotations between these ephemerides. 


\section{Acknowledgments}

We are grateful to Drs R.W.Hellings, D.C.Backer for the donation of the earlier timing data of PSR1937+214 used in this analysis, to Dr. J.Taylor for the permission to use his latest data and results and to Drs. P.Bretagnon and J.F.Chandler for helpful discussions.

\section{Bibliography}

Backer D.C., Kulkarni S.R., Heiles C., Davis M.M. \& Goss W.M., (1982), Nature, 300, p.615.

Backer D.C., Fomalont E.B., Goss W.M., Taylor J.H. \& Weisberg J.M., (1985), Astron. J., 90, p.2275.

Bartel N., Capallo R.J., Ratner M.I.,Rogers A.E.E., Shapiro I.I. \& Whitney A.R., (1985), Astron. J., 90, p.318.

Bevington P.R., (1969), Data Reduction and Error Analysis for the Physical Sciences, McGraw-Hill Book Company.

Bretagnon P., (1982), Astron. \& Astrophys., 114, p278

Davis M.M., Taylor J.H., Weisberg J.M. \& Backer D.C., (1985), Nature, 315, p.547.

Fairhead L. \& Bretagnon P., (1989) accepted for publication by Astron. \& Astrophys.

Guinot B., (1986), Celestial Mech., 38, p.155.

Hellings R.W., (1986), Astron. J., 91, p.650.

Le Guyader C. (1988) in preparation

Murray C.A., (1989), Astron. \& Astrophys., 218, p.325

Rawley L.A., (1986), Ph.D. Thesis, Princeton.

Rawley L.A., Taylor J.H. \& Davis M.M., (1988), Astrophys. J, 326, p.947

Standish E.M., (1981), Astron. \& Astrophys., 101, p.L17

Standish E.M., (1982), Astron. \& Astrophys., 114, p.297

\section{Discussion}

MURRAY: (a) Were you not able to measure the parallax of PSR1937+21? (b) How far away do you think it is?

FAIRHEAD: The timing data from 1982 to 1984 was not precise enough to determine the parallax of PSR 1937+21. Parallax measur-ment from timing data should be possible using the most recent data. (b) PSR1937+21 is $2 \mathrm{kpc}$ away.

KOPEJKIN: I have two remarks.

(1) First, I think that you can delete in the right-hand-side of the equation for the timing model the term which depends on radial velocity of pulsar and one which depends on the transversal velocity quadratically. The radial velocity term is incorporated into the period of the pulsar and another term is incorporated into the pulsar's period derivative.

(2) As far as I understand, you consider TT scale as proper time at the geocenter, is that correct? (Author: Yes, it is). In my opinion it is better and more correct to consider the TT scale as the coordinate time of a geocentric reference system constructed by the procedure outlined in the previous report by Brumberg, Klioner and Kopejkin. Realization of TT is done on real surface of the Earth at the points of location of atomic clocks. More information about this question is contained in the paper of Brumberg and myself which will be published in the journal Celestial Mechanics. 